\title{
Estimating the outgoing longwave radiation from the FY-3B satellite visible infrared radiometer Channel 5 radiance observations
}

\author{
WU Xiao ${ }^{1,2 *} \&$ YAN JunJie ${ }^{1,2}$ \\ ${ }^{1}$ Key Laboratory of Radiometric Calibration and Validation for Environmental Satellites, China Meteorological Administration, Beijing 100081, China; \\ ${ }^{2}$ National Satellite Meteorological Center, Beijing 100081, China
}

Received February 15, 2011; accepted June 27, 2011; published online August 30, 2011

\begin{abstract}
Based on the infrared radiation transfer model, the outgoing longwave radiation (OLR) and Channel 5 radiance of Fengyun-3B (FY-3B) satellite visible infrared radiometer (VIRR) were simulated for 3812 global soundings. Using regression analysis of the simulations, an inverse model, which connected the flux equivalent brightness temperature with the channel brightness temperature, was derived. By applying the model to the FY-3B VIRR L1 data, the global OLR data at the time of the passing of the FY-3B were processed. The quality of the data was validated by comparing it with that of the NOAA-18 satellite's advanced very high resolution radiometer (AVHRR). The validation results show root mean square errors in the range $10-13 \mathrm{~W} / \mathrm{M}^{2}$ when comparing the daily average OLR of the VIRR with that of the NOAA-18 AVHRR, and the correlation coefficients were in the range 0.97-0.98. The larger RMSE is mainly due to the different passing times of the two satellites for the specific locations on the Earth. An example of the OLR data and its preliminary applications are given.
\end{abstract}

OLR, infrared radiation transfer, inverse model, regression analysis, validation

Citation: Wu X, Yan J J. Estimating the outgoing longwave radiation from the FY-3B satellite visible infrared radiometer Channel 5 radiance observations. Chinese Sci Bull, 2011, 56: 3480-3485, doi: 10.1007/s11434-011-4686-6

The visible infrared radiometer (VIRR) on board the Fengyun-3B (FY-3B) polar orbiting meteorological satellite is an instrument similar to the National Oceanic and Atmospheric Administration (NOAA) satellite's advanced very high resolution radiometer (AVHRR). It has 10 channels to observe the shortwave and longwave radiations reflected and emitted by the Earth's atmospheric system. The split window, Channels 4 and 5, of the VIRR, are similar to that of the AVHRR. The spectrums of the VIRR are listed in Table 1. Traditionally the brightness temperature of the AVHRR Channel 5 has been adopted to calculate the outgoing longwave radiation flux (OLR) at the outer extremes of the atmosphere through the equation [1]: $T_{F}=A+B \times T_{B 5}+C \times$ $T_{B 5}^{2}$. In the NOAA National Environmental Satellite, Data, and Information Service (NESDIS), the equation has been used to produce OLR data for more than 30 years. OLR data

*Corresponding author (email: wuxiao@cma.gov.cn) have been widely studied by scientists in climate models, predictions and diagnoses. In November 2010, China successfully launched the FY-3B polar orbiting meteorological satellite and the satellite ground application system began its operation in February 2011. Subsequently, data from the FY-3B [2] have been archived and distributed on the web. The OLR data are some of the data available.

\section{Developing the OLR inverse model of VIRR}

The development of the OLR inverse model for the VIRR was based on infrared radiation transfer simulations [3-5] for a large number of atmospheric soundings and regression analysis. First, the OLR and Channel 5 radiance of VIRR were simulated for each sounding profile. Then a regression equation was derived by statistically analyzing the simulated results. Figure 1 shows our flowchart for developing the 
Table 1 Spectrums of VIRR

\begin{tabular}{cc||cc}
\hline Channel & Bandwidth $(\mu \mathrm{m})$ & Channel & Bandwidth $(\mu \mathrm{m})$ \\
\hline 1 & $0.58-0.68$ & 6 & $1.58-1.64$ \\
2 & $0.84-0.89$ & 7 & $0.43-0.48$ \\
3 & $3.55-3.95$ & 8 & $0.48-0.53$ \\
4 & $10.3-11.3$ & 9 & $0.53-0.58$ \\
5 & $11.5-12.5$ & 10 & $0.90-0.965$ \\
\hline
\end{tabular}

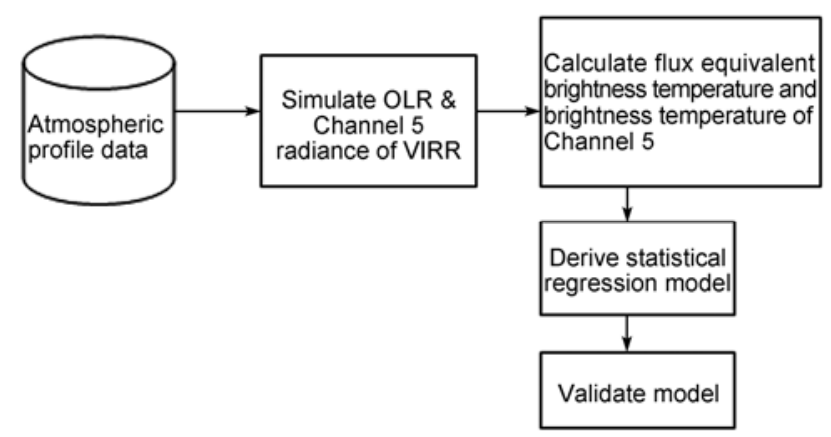

Figure 1 Flowchart of the process for establishing the OLR inverse model of VIRR.

inverse model.

\subsection{Calculation of the radiance at the top of the atmosphere}

In total 3812 atmospheric profiles representing various weather conditions encountered on the Earth were included in the calculations. An infrared radiation transfer equation $[6,7]$ was chosen to calculate the radiance at the top of each profile. The equation used is

$$
I(v, T, \theta)=B\left(v, T_{\mathrm{S}}\right) \times \tau_{\mathrm{S}}(\theta)+\int_{\tau_{\mathrm{S}}}^{1} B(v, T) \mathrm{d} \tau .
$$

The discrete equation of the above is

$$
I(v, T, \theta)=B\left(v, T_{\mathrm{S}}\right) \times \tau_{\mathrm{S}}(\theta)+\sum_{i=0}^{40} B\left(v, T_{i}\right) \Delta \tau_{i} .
$$

In overcast conditions, cloud is treated as a blackbody, so the radiance at the top of the atmosphere is

$$
I(v, T, \theta)=B\left(v, T_{\mathrm{C}}\right) \times \tau_{\mathrm{C}}(\theta)+\sum_{i=0}^{N} B\left(v, T_{i}\right) \Delta \tau_{i} .
$$

Here $I(v, T, \theta)$ is the radiance at the top of the atmosphere, $T_{\mathrm{S}}$ is the surface temperature, $\tau$ is the monochromatic transmittance, $v$ is the wavenumber, $\theta$ is the local zenith angle, $T_{i}$ is the air temperature of layer $i, \Delta \tau_{i}=\tau_{i+1}-\tau_{i}, B$ is the Plank Function, $T_{\mathrm{C}}$ is the temperature of the cloud top, $N$ is the layer number from the cloud top to the atmospheric top. The Plank Function $B$ is

$$
B(v, T)=\frac{c_{1} v^{3}}{\mathrm{e}^{c_{2} v / T}-1.0},
$$

where $c_{1}=1.191066 \times 10^{-5}, c_{2}=1.438681$, and $T$ is the air temperature.

\subsection{Calculation of the OLR and flux equivalent brightness temperature}

The total intensity of each profile is calculated using

$$
I(\theta)=\sum_{i=1}^{280} I\left(v_{i}, T, \theta\right) \Delta v
$$

Here the radiances in wavenumber $0-2800 \mathrm{~cm}^{-1}$ are summed, $\Delta v=10 \mathrm{~cm}^{-1}$. The outgoing longwave radiation flux $E$ (also OLR) [7] is written as

$$
E=2 \pi \int_{0}^{\frac{\pi}{2}} I(\theta) \sin \theta \cos \theta \mathrm{d} \theta
$$

The flux equivalent brightness temperature is then calculated by

$$
T_{\mathrm{F}}=\left(\frac{E}{\sigma}\right)^{\frac{1}{4}}
$$

where $\sigma$ is the Sdefan-Boltzmann constant, $\sigma=5.6693 \times$ $10^{-8}$, and $T_{\mathrm{F}}$ is the flux equivalent brightness temperature.

\subsection{Simulating the channel 5 radiance of VIRR}

The VIRR Channel 5 radiance is simulated by the following equation:

$$
I\left(v_{0}, T, \theta\right)=\frac{\int_{\gamma_{1}}^{\gamma_{2}} I(v, T, \theta) f(v) \mathrm{d} v}{\int_{\gamma_{1}}^{\gamma_{2}} f(v) \mathrm{d} v} .
$$

Here $v_{0}$ is the channel central wavenumber, $f(v)$ is the spectral response function, and $I\left(v_{0}, T, \theta\right)$ is the Channel 5 radiance. The radiance has the following limb darkening relationship:

$$
\begin{aligned}
I\left(v_{0}, T, 0\right)= & {\left[1+\alpha_{2}(\sec \theta-1)+\beta_{2}(\sec \theta-1)^{2}\right] } \\
& \times I\left(v_{0}, T, \theta\right)+\alpha_{1}(\sec \theta-1)+\beta_{1}(\sec \theta-1)^{2},
\end{aligned}
$$

where $I\left(v_{0}, T, 0\right)$ is the radiance observed at nadir, and $\alpha_{1}$, $\alpha_{2}, \beta_{1}, \beta_{2}$ are regression coefficients. The brightness temperature of Channel 5 is then calculated as

$$
T_{B 5}=\frac{c_{2} v_{0}}{\ln \left(c_{1} v_{0}^{3} / I\left(v_{0}, T, 0\right)+1.0\right)} .
$$

Here $c_{1}, c_{2}$ are the Plank constants, and $T_{B 5}$ is the bright- 
ness temperature of Channel 5.

\subsection{Analyzing the statistical relationship of $T_{\mathrm{F}}$ and $T_{B 5}$}

Based on the simulated $T_{B 5}$ and $T_{\mathrm{F}}$ for 3812 soundings, a scatterplot of $T_{B 5}$ and $T_{\mathrm{F}}$ was plotted. Figure 2 shows the scatterplot. The the statistical relationship between the two was then obtained by applying least-squares regression analysis to the simulated $T_{B 5}$ and $T_{\mathrm{F}}$ data. The relationship is of the form:

$$
T_{\mathrm{F}}=A+B \times T_{B 5}+C \times T_{B 5}^{2},
$$

where $A, B$, and $C$ are regression coefficients.

\section{OLR calculation method}

The OLR was calculated using VIRR L1 data and the regression equation. The detailed procedure follows.

\subsection{Calculating the radiance of VIRR Channel 5}

A calibration equation is used to convert the digital number of VIRR Channel 5 into radiance and is given by

$$
R(\theta)=s \times I_{5}+d,
$$

where $R(\theta)$ is the radiance of VIRR Channel 5, $s$ and $d$ are calibration coefficients of Channel $5, I_{5}$ is the digital number of Channel 5.

\subsection{Limb darkening correction}

The radiance observed at satellite zenith angle $\theta$ is corrected to the nadir radiance through the limb darkening equation:

$$
\begin{aligned}
R(0)= & {\left[1+\alpha_{2}(\sec \theta-1)+\beta_{2}(\sec \theta-1)^{2}\right] } \\
& \times R(\theta)+\alpha_{1}(\sec \theta-1)+\beta_{1}(\sec \theta-1)^{2} .
\end{aligned}
$$

Here $R(0)$ is the nadir radiance, $\alpha_{1}=-5.62987, \alpha_{2}=$ $0.08599, \beta_{1}=0.31874, \beta_{2}=-0.00447$.

\subsection{Calculating the brightness temperature of VIRR} Channel 5

The brightness temperature of Channel 5 is calculated using the inverse Plank Function:

$$
T_{B 5}=\frac{c_{2} v_{0}}{\ln \left(c_{1} v_{0}^{3} / R(0)+1\right)},
$$

where $c_{1}=1.191065 \times 10^{-5}, c_{2}=1.438681, v_{0}=856.50 \mathrm{~cm}^{-1}$, the unit of $T_{B 5}$ is $\mathrm{K}$.

\subsection{Calculating the flux equivalent brightness temperature}

The flux equivalent brightness temperature is obtained through the regression equation:

$$
T_{\mathrm{F}}=A+B \times T_{B 5}+C \times T_{B 5}^{2} .
$$

Here $A=10.50007, B=1.13333$, and $C=-0.000917$.

\subsection{Calculating the OLR}

The OLR flux is calculated by the equation

$$
E=\sigma \times T_{\mathrm{F}}^{4}
$$

Here $\sigma=5.6693 \times 10^{-8}, E$ is OLR, and the unit of $E$ is $\mathrm{W} / \mathrm{M}^{2}$.

\subsection{Calculation of the daily mean OLR}

In simple terms, the daily average OLR is the mean of the two values obtained by satellite day time and night time observations, and the equation is

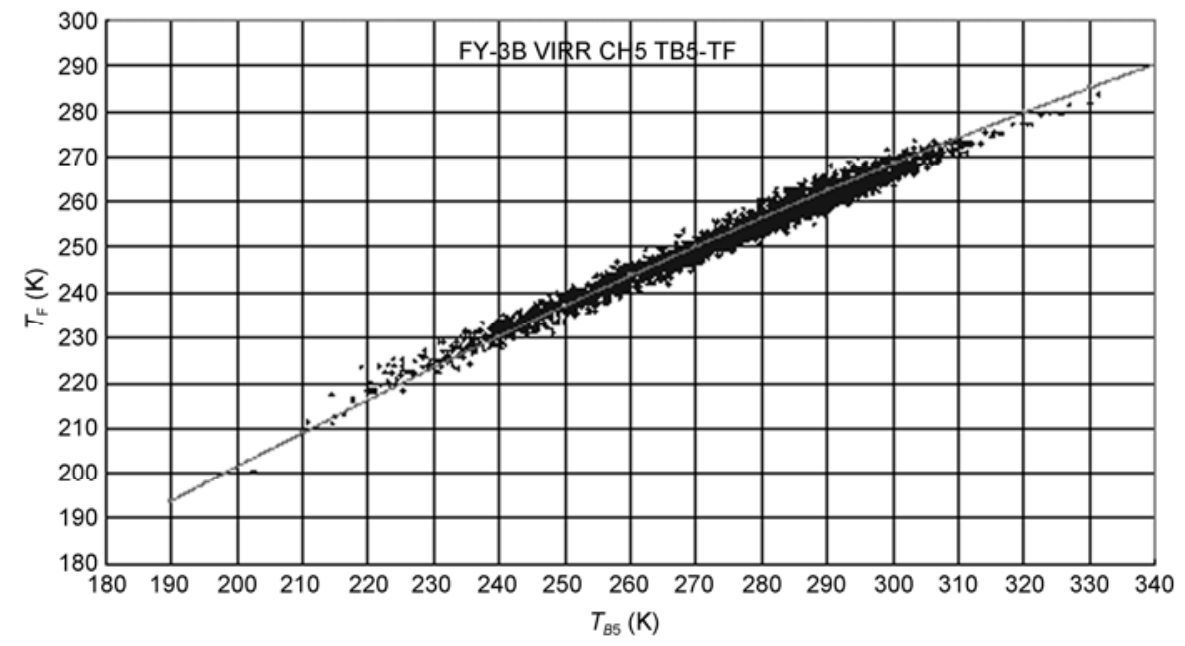

Figure 2 Scatterplot of the flux equivalent brightness temperature and the brightness temperature of VIRR Channel 5. 


$$
\operatorname{OLRM}=\frac{E_{\mathrm{D}}+E_{\mathrm{N}}}{2}
$$

Here $E_{\mathrm{D}}, E_{\mathrm{N}}$ are the OLR fluxes at the daytime and nighttime of satellite observations.

\section{The OLR product of VIRR and its quality}

The OLR data of the VIRR are the grid data which cover the global area with a data resolution of $0.01 \times 0.01$ in latitude and longitude. They are composed of six different files which are the daytime, nighttime, daily average, 5-day average, 10-day average, and monthly average OLR grid files. Figure 3 shows an example of the daily average OLR data. Because FY-3B and NOAA-18 have nearly the same overpass time, and the calculation method for the OLR is similar, we chose the NOAA-18 OLR data to validate the data of the VIRR.

By analyzing the isoline diagrams of the two and calcu- lating the root mean square errors (RMSE) and correlation coefficients, the quality of the VIRR data is tested. Figures 4 and 5 show the isoline diagrams of the FY-3B VIRR OLR and NOAA-18 AVHRR OLR. The RMSE in Figures 4 and 5 is $10.28 \mathrm{~W} / \mathrm{m}^{2}$, and the correlation coefficient is 0.9733. Many factors contribute to a large RMSE. The main factor is the different passing time of the two satellites for a specific location on the earth. Another is the accuracy of the instrument calibration. For the VIRR, the temperature of Channel 5 is $0.7 \mathrm{~K}$ less than that of the AVHRR for $T_{B 5}>290 \mathrm{~K}$, and the OLR flux is $2 \mathrm{~W} / \mathrm{m}^{2}$ less than that of the AVHRR for OLR $>270 \mathrm{~W} / \mathrm{m}^{2}$.

\section{Preliminary applications}

The OLR is an important parameter of the energy balance for the earth's atmospheric system. It is a necessary input of climate models. Because an OLR $\geqslant 250 \mathrm{~W} / \mathrm{m}^{2}$ represents the area dominated by subtropical anticyclones well, and an

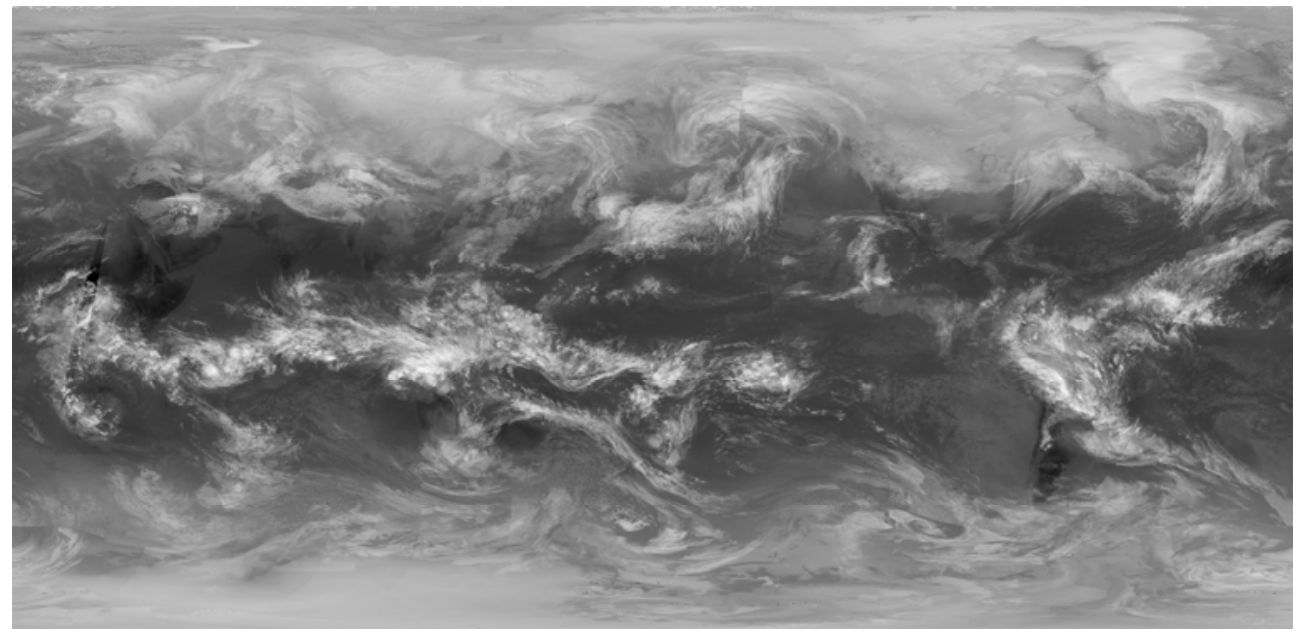

Figure 3 Grayscale image of the global daily average OLR data of the FY-3B VIRR (Feb. 8, 2011).

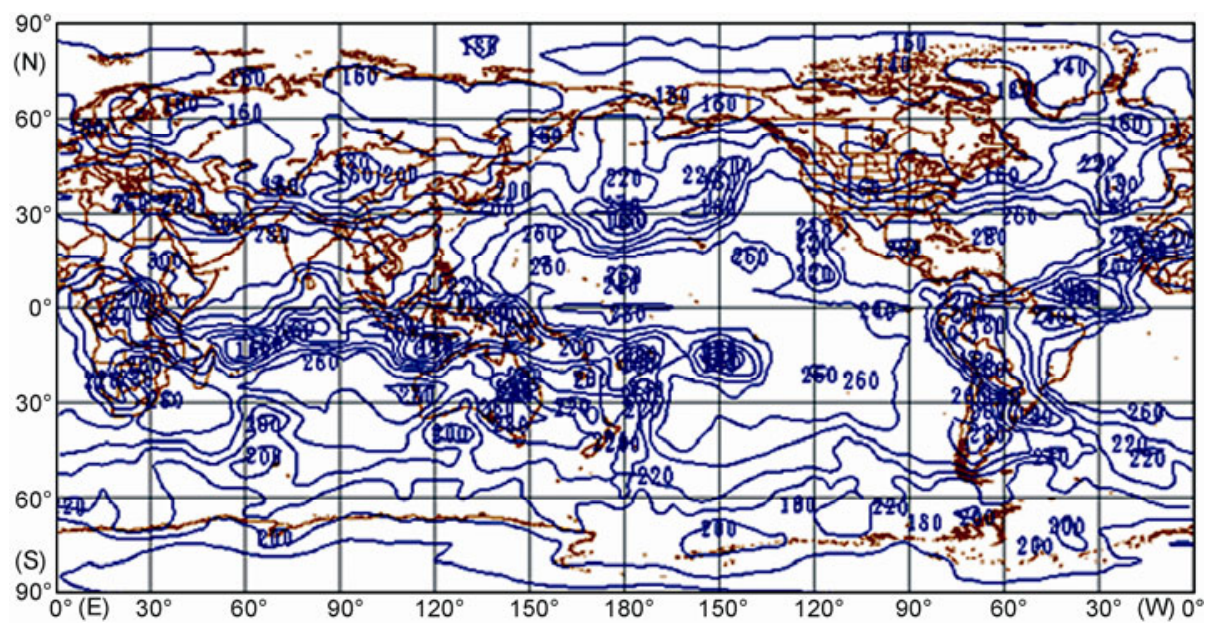

Figure 4 Isoline diagram of the daily average OLR for the FY-3B VIRR (Feb. 8, 2011, Unit: W/m²). 


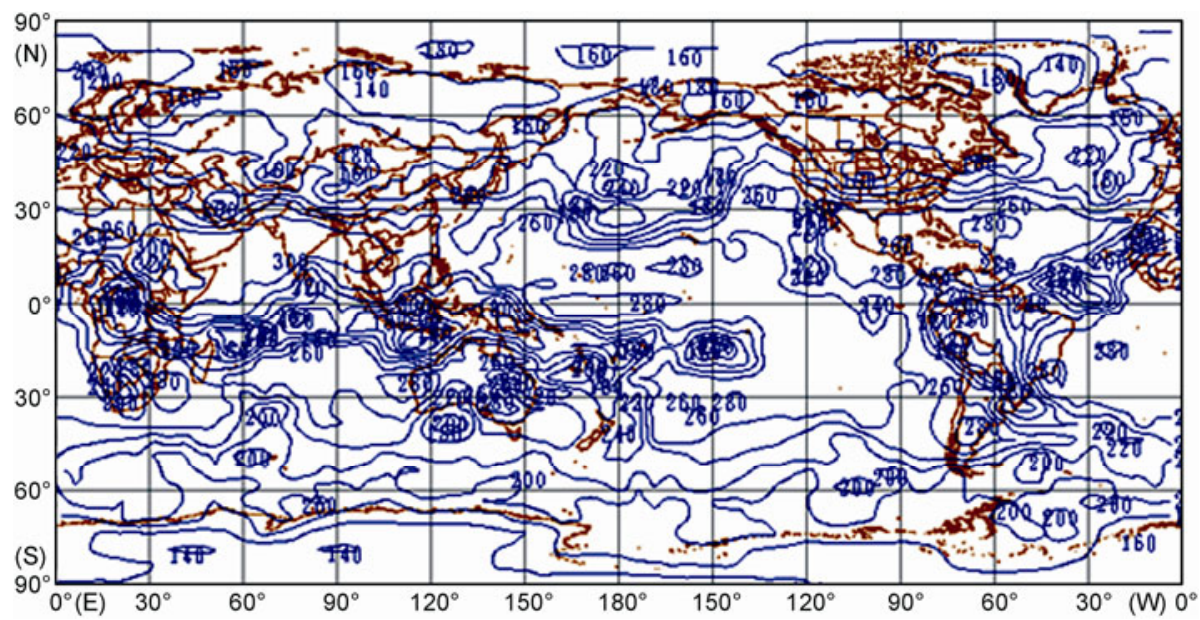

Figure 5 Isoline diagram of the daily average OLR for the NOAA-18 AVHRR (Feb.8, 2011).
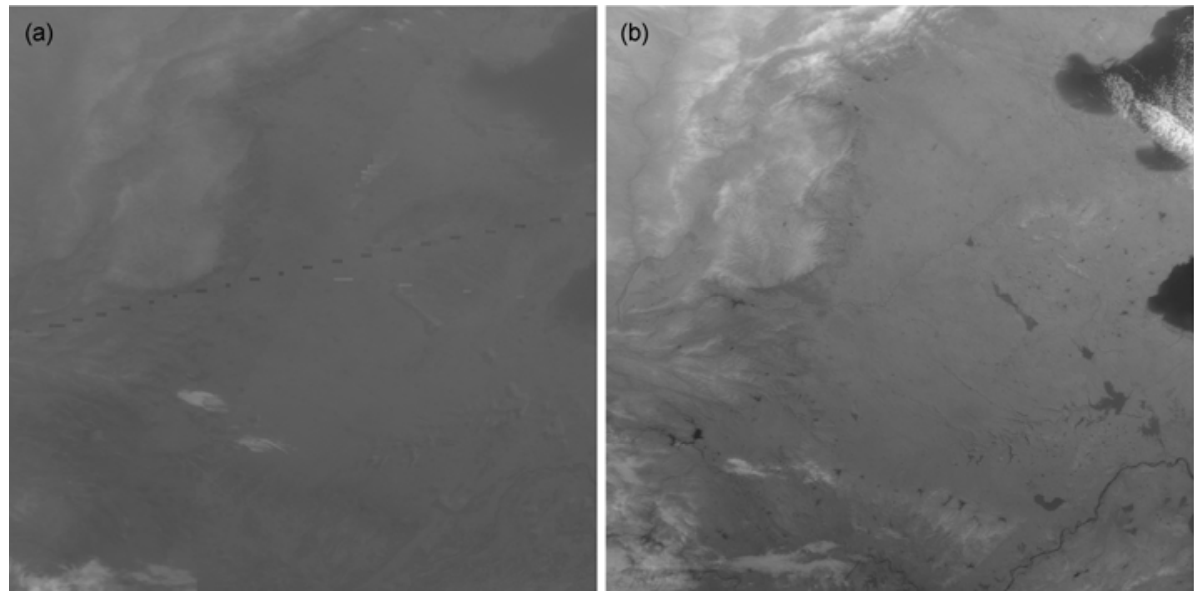

Figure 6 Grayscale images of the OLR data $\left(30^{\circ}-40^{\circ} \mathrm{N}, 110^{\circ}-120^{\circ} \mathrm{E}\right)$. (a) Dec. 1, 2008; (b) Dec. 5, 2008 (RES $\left.=0.01 \times 0.01\right)$.

OLR $\leqslant 220 \mathrm{~W} / \mathrm{m}^{2}$ reflects the axis location of the Inter tropical Convergence Zone, OLR data have been used in climate prediction. In the national climate center of China, OLR data from the NOAA AVHRR have long been used in monitoring the monsoon of the South China Sea. Many scientists use OLR data derived from different satellites to analyze the summer subtropical anticyclone in China [8,9]. Figure 3 shows that the grey image of the OLR is similar to a cloudy image which reflects the clear and cloudy conditions of the underlying surface. Therefore it can be used to directly analyze drought and flood weather processes. Cold wave weather is also well reflected in an OLR image. Figure 6(a) and (b) is two grayscale images of the daily average OLR data from the FY-3A in North China on Dec. 1, 2008 and Dec. 5, 2008, respectively. The dark color in Figure 6(a) represents the high value of the OLR and the warm weather before the cold wave. The brighter color in Figure 6(b) represents the low value of the OLR caused by the drastic decreasing temperature of the underlying surface during the process. Figure 7 shows the deviation of 2 days of OLR readings. Moreover, because of the high correlation between

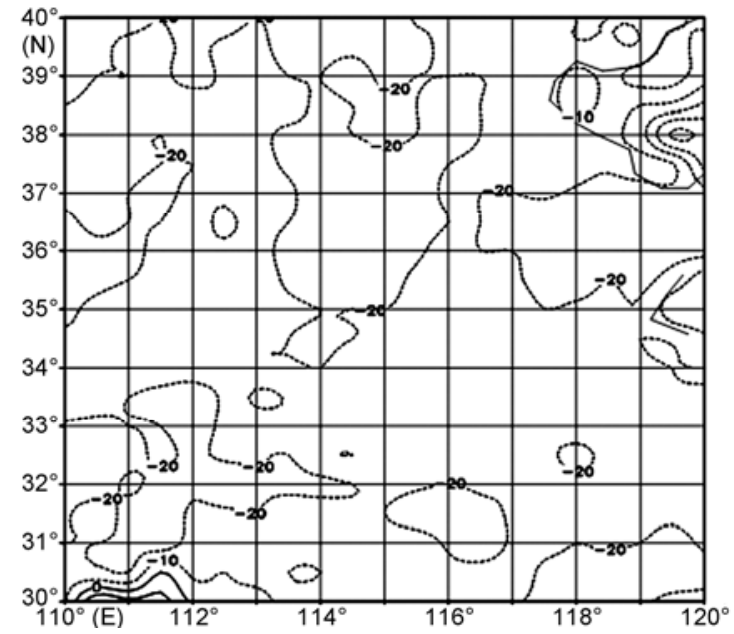

Figure 7 Isoline diagram of the daily average OLR deviation between Dec. 1 and 5, 2008.

cloud precipitation and the OLR, the data have been adopted to estimate the amount of precipitation in the United States $[10,11]$. 


\section{Conclusions}

This paper presents the development of an inverse model for calculating the OLR using the VIRR Channel 5 brightness temperature, and the method of estimating the OLR using VIRR L1 data. We also discuss the validation of the OLR data and can see that there is still much work to be done to improve the quality of the data. One approach to improving the quality is to change the content of the absorption gases in the infrared radiation transfer simulation using the newest content data, such as that of $\mathrm{CO}_{2}, \mathrm{CH}_{4}$ and $\mathrm{N}_{2} \mathrm{O}$. Another is to adopt the newest soundings in the simulation calculation. Finally improvement in instrument calibration is also important to enhance the quality of OLR data.

1 Ohring G, Gruber A, Ellingson R. Satellite determinations of the relationship between total longwave radiation flux and infrared window radiance. J Appl Meteorol, 1984,123: 416-425

2 Huang F X, Liu N Q, Zhao M X, et al. Vertical ozone profiles deduced from measurements of SBUS on FY-3 satellite. Chinese Sci
Bull, 2010, 55: 943-948

3 Wu X. Calculation of earth atmospheric transmissivity and radiance (in Chinese). J Appl Meteorol Sci, 1998, 9: 124-128

4 Selby J E A, McClatchey R A. Atmospheric transmittance from 0.25-28.5 $\mu \mathrm{m}$ : Computer code LOWTRAN-2. AFCRL Technical Report, TR-73-0745. 1973

5 Kneizys F Y, Shettle E P, Gallery W O. Atmospheric transmittance and radiance: The LOWTRAN 5 Code. In: Atmospheric Transmission, Proceedings of the Meeting, Bellingham WA: SPIE-the International Society for Optical Engineering, 1981. 116-124

6 Peixoto J P, Oort A H. Physics of Climate (in Chinese). Beijing: Meteorological Press, 1995. 72-103

7 Wu X. OLR inverse model for geostationary meteorological satellite FY-2D (in Chinese). Meteorol Sci Tech, 2008, 36: 634-638

8 Jang S C, Dai Z Y. The climate characteristics of west pacific subtropical high observed by satellites (in Chinese). Chinese Sci Bull, 1989, 19: 1492-1493

9 Xu C H, Ni Y Q, Zhao F K. A method for describing west pacific subtropical high by OLR data (in Chinese). J Appl Meteorol Sci, 2001, 12: 378-382

10 Xie P P, Arkin P A. Global monthly precipitation estimates from satellite-observed outgoing longwave radiation. J Clim, 1998, 11: 137-164

11 Arkin P A, Kelkar R R. Large-scale precipitation and outgoing longwave radiation from INSAT-1B during the 1986 southwest monsoon season. J Clim, 1989, 2: 619-628

Open Access This article is distributed under the terms of the Creative Commons Attribution License which permits any use, distribution, and reproduction in any medium, provided the original author(s) and source are credited. 